\title{
Major depression in temporal lobe epilepsy with hippocampal sclerosis: clinical and imaging correlates
}

\section{Regula S Briellmann, Malcolm J Hopwood, Graeme D Jackson}

See end of article for authors' affiliations

Correspondence to: Professor Graeme D Jackson, Brain Research Institute, Neurosciences Building, Austin Health, Heidelberg West, Victoria 3081, Australia; BRI@brain.org.au

Received 18 August 2006 Revised 3 January 2007 Accepted 4 January 2007 Published Online First 26 January 2007
Purpose: Refractory temporal lobe epilepsy (TLE) is often associated with hippocampal sclerosis (HS). Patients with major depression (MD) may also show structural abnormalities in the limbic system. Co-occurrence of TLE with HS and MD is not uncommon. We have investigated the clinical and morphological characteristics of TLE patients in relation to MD.

Methods: 34 TLE patients with HS were assessed at a Comprehensive Epilepsy Programme. All relevant clinical data were obtained, including the history of antecedent events to epilepsy. MD was diagnosed based on detailed psychiatric investigation. MRI was used to measure the volume and tissue signal (T2 relaxometry) of the hippocampus and amygdala. The imaging data were expressed as a percentage of the values obtained in a series of 55 controls.

Results: A history of MD was present in $15(44 \%)$ of 34 patients. Patients with MD had a longer duration of their epilepsy $(p<0.05)$ and a lower frequency of antecedent events $(13 \%$ with $M D, 58 \%$ without $M D$, $p<0.05$ ). Both groups had a similar degree of ipsilateral HS (small hippocampal volume, increased hippocampal T2 relaxation time) and demonstrated bilateral amygdaloid atrophy. However, the contralateral amygdala showed lower signal in the presence of MD (97 (9) ms; no MD 103 (8) ms; ANCOVA, p=0.02). Conclusion: The integrity of the amygdala may influence mood disturbances in TLE patients with HS, as depression was associated with a relative preservation of the contralateral amygdala. In contrast, hippocampal abnormalities were not related to the presence of depression.
$\mathrm{R}$ efractory temporal lobe epilepsy (TLE) is a chronic disease, associated with a major social burden reflected in reduced quality of life. Refractory TLE is often associated with mood disorders, particularly with depression. The incidence of depressive disorders in patients with epilepsy has been estimated to range between $30 \%$ and $70 \%,{ }^{12}$ which indicates that depression is the most frequent comorbidity of epilepsy. It has also been suggested that the presence of depression has an even more negative effect on the quality of life than seizure frequency. ${ }^{3}$ The clinical presentations of depressive disorders in epilepsy can be identical to those in non-epileptic patients and can include major depression, bipolar and dysthymic disorders, and minor depression. ${ }^{4}$ Kanner suggested that the relationship between epilepsy and depression is "bidirectional" with the presence of a common pathogenic process facilitating the occurrence of one disease in the presence of the other. ${ }^{4}$ Supportive of this model is that similar structures of the limbic network are involved in both diseases. In a single photon emission computerised tomography study, it was demonstrated that depressed epilepsy patients show relatively increased blood flow in the limbic system compared with non-depressed patients, ${ }^{5}$ supporting the importance of the limbic system in depression associated with epilepsy. The hippocampus and the amygdala are the prominent mesial temporal structures of the limbic network, and may have significant roles in this "bidirectional" relationship.

Recent research on patients with depression alone has suggested structural as well as functional changes in the limbic network. Several studies have demonstrated a reduction in hippocampal volume in patients with major depression, ${ }^{6-8}$ as well as with post-traumatic stress disorder. ' Whereas the hippocampus is primarily involved in memory consolidation, ${ }^{10}$ the amygdala has been associated with the processing of emotions, particularly negative emotions. ${ }^{11}$ The amygdala appears to undergo functional alterations, ${ }^{12}{ }^{13}$ as well as structural changes ${ }^{12}{ }^{14}$ in patients with depression. However, there is some controversy in the literature on the direction of the volumetric changes of the amygdala. ${ }^{15}$ The function of the amygdala in the interpretation of emotional meanings may be altered in depression. ${ }^{16}$

On the other hand, mesial temporal abnormalities are the most common single abnormality associated with TLE. ${ }^{17}$ Hippocampal sclerosis (HS) is the most common lesion in patients with TLE, refractory to antiepileptic medication. Approximately $60 \%$ of HS patients have a history of a precipitating injury, such as prolonged febrile convulsions in early childhood. These antecedent events to the development of TLE are thought to induce an epileptogenic lesion in the hippocampus. ${ }^{18}$ These observations support the fact that HS is an acquired lesion. ${ }^{19}$ Hippocampal sclerosis can be diagnosed with magnetic resonance imaging. ${ }^{20}$ The reduction in hippocampal volume and the increase in the T2 weighted signal intensity can be quantified. ${ }^{21}$ Patients with antecedent events have pronounced hippocampal abnormalities compared with TLE patients in whom no antecedents have been documented. ${ }^{22}$

Despite the known relationship between TLE and depression, only a few studies have investigated whether patients with this comorbidity display a characteristic pattern of clinical and imaging findings, distinguished from patients with isolated TLE. Several studies demonstrated altered morphology of the mesial temporal structures in TLE patients with associated aggressive behaviour ${ }^{23}$ or psychosis, ${ }^{24}{ }^{25}$ whereas others have investigated the predictive value of clinical epilepsy characteristics, such as seizure focus lateralisation, on the co-occurrence of depression in TLE. ${ }^{26}$ However, the relationship between clinical and morphological characteristics on the co-occurrence

Abbreviations: CEP, Comprehensive Epilepsy Programme; CPS, complex partial seizures; GTCS, generalised tonic-clonic seizures; HS, hippocampal sclerosis; MD, major depression; TLE, temporal lobe epilepsy 
of TLE and depression has not been investigated to date. We aim to assess whether TLE patients with associated major depression have a different pattern of hippocampal and amygdaloid damage, and of clinical epilepsy characteristics, than TLE patients without such mood disorders.

\section{METHODS \\ Patients}

We studied 34 consecutive patients with refractory TLE associated with HS that had detailed psychiatric assessment as part of their presurgical epilepsy evaluation between January 2003 and October 2004. All patients were assessed in the Comprehensive Epilepsy Programme (CEP) of Austin Health, Melbourne, Australia. These investigations included clinical characterisation, video-EEG telemetry, psychiatric and neuropsychological assessment, MRI and, in the majority of the cases, positron emission tomography as well as ictal and interictal single photon emission tomography. The diagnosis of HS was based on an epilepsy dedicated $1.5 \mathrm{~T}$ magnetic resonance protocol using established criteria. ${ }^{27}$ The diagnosis of TLE was based on the decision of the CEP meeting, which takes all available information from all investigations into account and formulates a recommendation for epilepsy surgery. The ipsilateral side indicates the side of the seizure focus. Significant antecedent events included severe perinatal complications, severe postnatal head injury, significant cerebral infection, stroke and complicated febrile seizures, as previously described by our group. ${ }^{28}$ All other risk factors (eg, prematurity, uncomplicated febrile seizures) were not regarded as significant. Age at onset of epilepsy was defined as the age at the first habitual seizure. Seizure characteristics were expressed as frequency of complex partial seizures (CPS) and occurrence of generalised tonic-clonic seizures (GTCS). The number of CPS per week was counted based on seizure diaries kept during the 3 months leading up to the inpatient monitoring (CEP). Patients and their cares received appropriate instructions on how to keep the diary in their outpatient visits. The presence or absence of GTCS as part of seizure semiology was identified based on patient histories.

\section{Diagnosis of depression}

The psychiatric assessment was performed routinely in all patients as part of the presurgical investigation of the CEP programme. It was conducted in all subjects by a consultant neuropsychiatrist, experienced in the assessment of psychiatric aspects of epilepsy ( $\mathrm{MH})$. This psychiatric evaluation was based on an approximately hour long semistructured interview, providing diagnosis according to DSM-IV criteria (APA, 1994). The interview was summarised and the diagnosis recorded in the patient's file at the time of the investigation. For the purpose of this study, $\mathrm{MH}$ also performed a retrospective file audit of all subjects in order to maximise the classification of psychiatric symptoms. The TLE patients were classified as having the comorbidity major depression (MD) when they had either current MD or had suffered from MD in the past. Past or current use of antidepressants was noted. Minor depressive mood swings were not considered as qualifying for the diagnosis. Patients with psychotic disorders, or suffering from other psychiatric diseases, were not included in the study.

The study was approved by the Austin Health Human Research Ethics Committee, and all subjects gave informed consent to participate.

\section{MR experiments}

Research MR imaging was performed on a 3 T GE LX Horizon scanner (Milwaukee, Wisconsin, USA). For the volumetric analysis, we acquired $\mathrm{Tl}$ weighted images using a fast spoiled gradient recalled echo at steady state (FSPGR) sequence (TR/TE $8.9 / 1.9$, flip angle 20 , matrix size $256 \times 256$ and a field of view of $25 \times 18.75 \mathrm{~cm}$ ) with contiguous coronal slices of $1.5 \mathrm{~mm}$ thickness. For the T2 relaxometry analysis, we used a CarrPurcell-Meiboom-Gill sequence with eight images per location at echo times between $28 \mathrm{~ms}$ and $231 \mathrm{~ms}$ (TR $2080 \mathrm{~ms}, 8$ echoes per location, $256 \times 128$ matrix, 1 NEX, $24 \mathrm{~cm}$ field of view, 24 slices, $5 \mathrm{~mm}$ thick, no gap) with an acquisition time of $10 \mathrm{~min}$. The images were acquired perpendicular to the long axis of the hippocampus.

\section{Measurements}

Volumetric measurements were performed using NIH image software (NIH-image 1.63, available at http://rsb.info.nih.gov/ nih-image). Measurement of hippocampal, amygdaloid and whole brain volumes used previously described methods. ${ }^{29}$ Standard anatomical atlases and relevant previous publications were used as guidelines for the hippocampal and amygdaloid measurements. ${ }^{30}$ Hippocampi and the amygdalae were manually outlined at each second slice, from posterior to anterior, and the slice volumes were summed.

The T2 relaxation times were measured with Functool (GE Medical Systems, Milwaukee, Wisconsin, USA). The analysis fits a single exponential to signal intensity values of corresponding pixels from each of the eight images per slice location. Measurement of the $\mathrm{T} 2$ relaxation times was achieved by placing a circular region of interest over a predefined area of anatomy, carefully avoiding inclusion of CSF. All measurements were performed bilaterally, using anatomical landmarks. We measured the T2 relaxation time bilaterally in the hippocampus and amygdala. In each subject, the region of interest comprised the maximal possible circular region within the boundaries of the structure, as previously described. ${ }^{21}$ This approach has been previously validated to give precise and reliable measurements for the most complex region of interest included, the hippocampus.

All measurements were performed blinded to the psychiatric diagnosis of the subjects. All values were expressed as ipsilateral and contralateral to the seizure focus. As normative data of volumetric measurements are gender dependent, all values were expressed as a percentage of corresponding values obtained from controls of the same gender, as described previously by our group. ${ }^{31}$ The control series consisted of 55 controls (mean age 34 (9) years, 29 men). These controls were free of any neurological or psychiatric disease, as based on a routine questionnaire, and had normal magnetic resonance imaging. The mean hippocampal volume of these controls was $2661(378) \mathrm{mm}^{3}$, the amygdaloid volume $1083(179) \mathrm{mm}^{3}$ and the brain volume $1184(135) \mathrm{cm}^{3}$. The T2 relaxation time was 90 (3) $\mathrm{ms}$ in the hippocampus and 84 (3) $\mathrm{ms}$ in the amygdala.

\section{Data analysis}

Differences in clinical and imaging findings were assessed between patients with and without MD. Continuous data were assessed using analysis of variance (ANCOVA), with the age at examination included as a covariate. For the volumetric analysis, the brain volume was added as a second covariate. Categorical data were assessed using $\chi^{2}$ tests (Fisher's exact $p$ value given). A p value $\leqslant 0.05$ was regarded as significant.

\section{RESULTS}

\section{Epilepsy characteristics and depression}

Of the 34 TLE-HS patients, 15 (44\%) had a diagnosis of MD. At the time of the investigation, five HS patients with MD displayed current symptoms of depression and nine were receiving antidepressants. Gender (15 males: seven with MD, 
Table 1 Clinical epilepsy characteristics in all patients

\begin{tabular}{|c|c|c|}
\hline & Major depression & No depression \\
\hline$n(\%)$ & $15(44)$ & $19(56)$ \\
\hline \multicolumn{3}{|l|}{ Gender (n (\%)) } \\
\hline Male & $7(47)$ & $8(42)$ \\
\hline Female & $8(53)$ & $11(58)$ \\
\hline Age at examination (y) & $42(10)$ & $35(10)$ \\
\hline Age at onset epilepsy (y) & $10(12)$ & $14(12)$ \\
\hline Duration epilepsy (y) & $31(17)$ & $21(12)^{*}$ \\
\hline \multirow{2}{*}{\multicolumn{3}{|c|}{ Presence of GTCS (\%) }} \\
\hline & & \\
\hline Present & $9(60)$ & $12(63)$ \\
\hline Absent & $6(40)$ & 7 (37) \\
\hline \multicolumn{3}{|l|}{ Side of seizure focus (\%) } \\
\hline Right & $3(20)$ & $7(37)$ \\
\hline Left & $12(80)$ & $12(63)$ \\
\hline \multicolumn{3}{|l|}{ Significant antecedents (\%) } \\
\hline Present & $2(13)$ & $11(58)^{*}$ \\
\hline Absent & $13(87)$ & $8(42)$ \\
\hline
\end{tabular}

CPS, complex partial seizures, measured per week over a 3 month observation period; GTCS, generalised tonic-clonic seizures, assessed as being part of the patient's seizure history or not.

Results shown are mean (SD) or frequency (\%).

Differences between patients with and without major depression were assessed.

ANCOVA (continuous variables) and $\chi^{2}$ tests (categorical variables). Patients with major depression had a longer duration of epilepsy and a lower incidence of antecedent events to the development of epilepsy. ${ }^{*}$ Significant results.

eight without MD) and seizure focus side (24 left HS: 12 with MD, 12 without MD) was not different between depressed and non-depressed HS patients (table 1). Age at examination, and age at the first habitual seizure did not differ between HS patients with and without MD. However, HS patients with MD had, on average, a 10 year longer duration of their epilepsy $(\mathrm{p}<0.05$, ANOVA). The frequency of significant antecedent events was reduced in depressed HS patients (2/15 patients with MD, 13\%) compared with non-depressed HS patients ( 11 / 19 patients without $\left.\mathrm{MD}, 58 \%, \chi^{2}, \mathrm{p}=0.01\right)$. There was no difference in the frequency of CPS per week (table 1). However, in both patient groups, seizure frequency showed large individual differences. Furthermore, there was no difference in the number of patients who had experienced GTCS; in both groups approximately $60 \%$ had occasional generalisation of their seizures.

\section{Structure of mesial temporal lobe and depression}

In refractory TLE patients, the volume of the ipsilateral hippocampus and amygdala was, on average, reduced by more than $25 \%$ of corresponding control values (table 2). The contralateral hippocampal volumes were only slightly reduced, whereas the contralateral amygdala showed atrophy of a similar degree as the ipsilateral amygdala. However, these volume deficits were not different between patients with and without MD. The ipsilateral hippocampal T2 relaxation time was markedly increased, again without a difference in relation to depression status (table 2). Interestingly, the T2 signal in the contralateral amygdala was increased in patients without depression (103 (8) ms) compared with patients with depression (97 (9) ms; ANCOVA, p=0.02).

\section{DISCUSSION}

Major depression was found in 44\% of refractory TLE patients, consistent with earlier studies on this topic. ${ }^{1}$ As expected, these TLE patients with a visual diagnosis of hippocampal sclerosis showed characteristic quantitative atrophy and signal increase in the ipsilateral mesial temporal structures. The degree of ipsilateral hippocampal damage did not predict the presence of
Table 2 Structural measurements in patients with hippocampal sclerosis in relation to mood state

\begin{tabular}{lll}
\hline & Major depression & No depression \\
\hline $\mathrm{n}$ Volumetrics & 15 & 19 \\
$\begin{array}{l}\text { Hippocampus } \\
\text { lpsi }\end{array}$ & & \\
$\quad$ Contra & $64(13)$ & $71(18)$ \\
$\begin{array}{c}\text { Amygdala } \\
\text { Ipsi }\end{array}$ & $93(18)$ & $97(17)$ \\
$\quad$ Contra & $76(19)$ & $76(19)$ \\
$\begin{array}{l}\text { T2 relaxometry } \\
\text { Hippocampus }\end{array}$ & $71(21)$ & $80(21)$ \\
$\quad$ lpsi & & \\
$\quad$ Contra & $117(15)$ & $120(10)$ \\
Amygdala & $102(7)$ & $102(6)$ \\
Ipsi & $102(15)$ & $105(9)$ \\
$\quad$ Contra & $97(10)$ & $103(8)^{*}$ \\
\hline
\end{tabular}

Volumes and T2 relaxation times are expressed as a percentage of normal values based on control values obtained in subjects of the same gender. Differences between depressed and non-depressed patients were assessed by ANCOVA, with age and total brain volume included as covariates for the volumetric measurements, and age for the T2 relaxation times.

*Only patients without depression had a contralateral T2 increase in the amygdala.

MD. This is in agreement with a recent study assessing hippocampal volumes in patients with HS in relation to depression scores. ${ }^{32}$ This study did not investigate $\mathrm{T} 2$ relaxation times or the degree of amygdaloid damage. Utilising these tools, we documented a relatively preserved contralateral amygdala in TLE patients with associated MD. Furthermore, a long duration of epilepsy and the absence of precipitating insults were associated with an increased risk of MD. There was no apparent association between seizure characteristics and the presence of MD. This may support the fact that depression is not merely a consequence of having (lots of) seizures, ${ }^{3}$ but may also reflect intrinsic difficulties in measuring seizure severity and their impact on the patient's mood.

For the purpose of our study, we combined patients with past and current symptoms of MD, as we aimed to assess whether certain structural abnormalities, commonly part of the spectrum of TLE with HS, would predispose to MD. Structural changes typically associated with HS are most commonly stable over time, and are postulated to be present early in the evolution of the epilepsy. ${ }^{17}$ On the other hand, both symptoms of depression and epilepsy vary over time, and it cannot be excluded that acute exacerbations could have an additional impact on the structure of the mesial temporal lobe. Our cross sectional study design did not allow us to assess these questions, but it can give information about an underlying difference between patients with TLE, with and without associated depression.

We documented a relative sparing of signal change in the contralateral amygdala in patients with TLE with associated MD. Contralateral mesiotemporal abnormalities have been described in patients with TLE. ${ }^{33-36}$ Volume deficits are typically contributed to neuronal cell loss, whereas an increase in T2 relaxation time, at least in the ipsilateral hippocampus, is thought to reflect gliosis. ${ }^{37}{ }^{38}$ Therefore, our findings could be interpreted as reflecting relatively less gliotic changes in the contralateral amygdala of patients with MD.

The second finding related to antecedent events. An antecedent event or an initial precipitating insult is a neurological event occurring prior to the onset of habitual seizures, and poses a risk for the development of epilepsy. ${ }^{19}$ Serial neuroimaging studies documented the evolution of HS after antecedent events. ${ }^{18} 39$ Antecedent events are typically 
found in up to $60 \%$ of refractory TLE patients, ${ }^{17}{ }^{40}$ but were much less frequent in our patients with MD. One could argue that these events may have been missed either at the time of their occurrence or when the patient was investigated for refractory epilepsy. However, our CEP pays careful attention in recording these events, and there is no reason to believe that this should have been different between the two patient groups. Alternatively, the aetiology of the epileptogenic lesion in patients with MD may be independent of antecedent events. Several studies have suggested that some TLE patients have developmental, prenatal structural abnormalities underlying their epilepsy, ${ }^{41}$ or a genetic predisposition for their seizures. ${ }^{42}$ However, the mechanisms of seizure focus generation in the absence of antecedent events are not well understood. ${ }^{42}{ }^{43}$ It is possible that this process is more focal and unilateral, as HS patients without antecedent events show generally less abnormality in the region of the seizure focus, and also less widespread damage to other ipsilateral and contralateral temporal lobe structures. ${ }^{22}$ One may argue that the amygdala shows less impairment because these patients were not exposed to initial precipitating events. So far, we have provided a potential explanation for the relationship between a low frequency of antecedent events and contralateral amygdaloid damage; it remains to be discussed whether these findings are related to $\mathrm{MD}$. On a psychological level, one could speculate that the absence of recognised antecedent events represents a higher risk for mood disorders, as these patients have no explanation of what might have caused their epilepsy. Epilepsy has a long history of misconceptions and social stigma, so the lack of a causative event may lead to a negative emotional reaction. Furthermore, the long duration of the disease may pose an additional burden to the patients, aggravating their mood disturbance. Alternatively, the absence of antecedent events may indicate a pre-existing abnormality of the limbic network, which may not only cause TLE but also predispose to depression. ${ }^{4}$ In patients with MD alone, histopathological studies found evidence of developmental disturbances in the limbic system. ${ }^{14}$

The importance of the amygdala volume in relation to psychiatric comorbidities has already been noted in previous studies on the integrity of the mesial temporal lobe. ${ }^{23} 44$ Particularly interesting in relation to our findings is that a relative preservation of the right amygdala was found in patients with refractory TLE and associated anxiety. ${ }^{44}$ As the majority of our patients had left HS, one could postulate a side specific involvement of the amygdala in mood disorders. The relative preservation of the contralateral amygdala may lead to increased or altered processing of negative emotions. Several studies have reported an increase in the metabolism of the amygdala in untreated MD when occurring as a sole disorder. ${ }^{12}{ }^{46}$ There is also indication for an alteration of the functional connectivity between the two amygdaloid nuclei in depressed patients. ${ }^{13} \mathrm{MD}$ has also been linked to a reduction in amygdaloid glial density, without an associated change in neuronal density. ${ }^{47}$ This finding mirrors our structural findings in depressed TLE patients, where the signal change, but not the volume change, showed an association with MD.

Depression as a sole disease is a clinically heterogenous condition. ${ }^{12}$ Depression associated with TLE is probably similarly heterogenous, lowering the power to detect differences related to depression. Structural and functional changes may be further altered during the course of the disease, with evidence for different findings during the first episodes, compared with chronic depression. ${ }^{7}$ The vast majority of our depressed patients have had more than one episode when examined, so our findings reflect a more chronic disease state. Furthermore, antidepressants may also alter the functional activity of the amygdala in particular. ${ }^{12}$ Some effects of depression may be reflected in changes in brain function, but not structure, and may thus not be detectable with the methods used in this study. Finally, for this study, we identified depression based on semistructured interviews, and combined the patients with past and current symptoms of MD into one single group. This approach may have affected our ability to detect abnormalities in brain structure, as it is conceivable that these abnormalities could be more pronounced in the acute stages of the disorder.

In conclusion, in the uncommon situation when TLE associated with HS develops without a precipitating event, there may be an increased risk of depression. A relatively preserved contralateral amygdala may lead to altered processing of negative emotions, and symptoms of depression may become clinically evident.

\section{ACKNOWLEDGEMENTS}

We wish to thank the patients for participating in this research study.

\section{Authors' affiliations \\ Regula S Briellmann, Graeme D Jackson, Brain Research Institute, Heidelberg, Melbourne, Australia \\ Malcolm J Hopwood, Brain Disorders Programme, Austin Health, Melbourne, Australia}

We are grateful to Neurosciences Victoria (NSV), the National Health and Medical Research Council (NHMRC) and the Brain Imaging Research Foundation Australia for financial support.

Competing interests: None.

\section{REFERENCES}

1 Prueter C, Norra C. Mood disorders and their treatment in patients with epilepsy. J Neuropsychiatry Clin Neurosci 2005;17:20-8.

2 Grabowska-Grzyb A, Jedrzejczak J, Naganska E, et al. Risk factors for depression in patients with epilepsy. Epilepsy Behav 2006;8:411-17.

3 Boylan LS, Flint LA, Labovitz DL, et al. Depression but not seizure frequency predicts quality of life in treatment-resistant epilepsy. Neurology 2004;62:258-61.

4 Kanner AM. Is major depression a neurologic disorder with psychiatric symptoms? Epilepsy Behav 2004;5:636-44.

5 Ring HA, Acton PD, Scull D, et al. Patterns of brain activity in patients with epilepsy and depression. Seizure 1999;8:390-7.

6 Bremner JD, Narayan M, Anderson ER, et al. Hippocampal volume reduction in major depression. Am J Psychiatry 2000;157:115-18.

7 Frodl T, Meisenzahl EM, Zetzsche T, et al. Hippocampal changes in patients with a first episode of major depression. Am J Psychiatry 2002;159:11112-18.

8 Mervaala E, Fohr J, Kononen M, et al. Quantitative MRI of the hippocampus and amygdala in severe depression. Psychol Med 2000;30:117-25.

9 Smith ME. Bilateral hippocampal volume reduction in adults with post-traumatic stress disorder: a meta-analysis of structural MRI studies. Hippocampus 2005; 15:798-807.

10 Cabeza R, Nyberg L. Imaging cognition II: An empirical review of 275 PET and fMRI studies. J Cogn Neurosci 2000;12:1-47.

11 Gur RE, McGrath C, Chan RM, et al. An fMRI study of facial emotion processing in patients with schizophrenia. Am J Psychiatry 2002;159:1992-9.

12 Drevets WC. Neuroimaging abnormalities in the amygdala in mood disorders. Ann N Y Acad Sci 2003;985:420-44.

13 Irwin W, Anderle MJ, Abercrombie HC, et al. Amygdalar interhemispheric functional connectivity differs between the non-depressed and depressed human brain. Neuroimage 2004;21:674-86.

14 Young KA, Holcomb LA, Yazdani U, et al. Elevated neuron number in the limbic thalamus in major depression. Am J Psychiatry 2004;161:1270-7.

15 Caetano SC, Hatch JP, Brambilla P, et al. Anatomical MRI study of hippocampus and amygdala in patients with current and remitted major depression. Psychiatry Res 2004; 132:141-7

16 Hastings RS, Parsey RV, Oquendo MA, et al. Volumetric analysis of the prefrontal cortex, amygdala, and hippocampus in major depression. Neuropsychopharmacology 2004;29:952-9.

17 Engel JJ, Williamson PD, Wieser H-G. Mesial temporal lobe epilepsy. In: Engel JJ, Pedley TA, eds. Epilepsy: a comprehensive textbook. Philadlephia: LippincottRaven, 1998:2417-26.

18 VanLandingham KE, Heinz ER, Cavazos JE, et al. Magnetic resonance imaging evidence of hippocampal injury after prolonged focal febrile convulsions. Ann Neurol 1998;43:413-26.

19 Falconer MA, Serafetinides EA, Corsellis JAN. Etiology and pathogenesis of temporal lobe epilepsy. Arch Neurol 1964;10:233-40. 
20 Berkovic SF, Andermann F, Olivier A, et al. Hippocampal sclerosis in temporal lobe epilepsy demonstrated by magnetic resonance imaging. Ann Neurol 1991;29:175-82.

21 Jackson GD, Berkovic SF, Duncan JS, et al. Optimizing the diagnosis of hippocampal sclerosis using magnetic resonance imaging. Am J Neuroradiol 1993; 14:753-62

22 Cendes F, Andermanmn F, Dubeau F, et al. Early childhood prolonged febrile convulsions, atrophy and sclerosis of mesial structures, and temporal lobe epilepsy: an MRI volumteric study. Neurology 1993:43:1083-7.

23 Tebartz van Elst L, Woermann FG, Lemieux L, et al. Amygdala enlargement in dysthymia - a volumetric study of patients with temporal lobe epilepsy. Biol Psychiatry 1999;46:1614-23.

24 Tebartz Van Elst L, Baeumer D, Lemieux L, et al. Amygdala pathology in psychosis of epilepsy: A magnetic resonance imaging study in patients with temporal lobe epilepsy. Brain 2002;125:140-9.

25 Briellmann RS, Kalnins RM, Hopwood MJ, et al. Postictal psychosis in TLE microdysplasia and anterior hippocampal preservation. Neurology 2000;55:1027-30.

26 Helmstaedter C, Sonntag-Dillender M, Hoppe C, et al. Depressed mood and memory impairment in temporal lobe epilepsy as a function of focus lateralization and localization. Epilepsy Behav 2004;5:696-701.

27 Briellmann RS, Pell GS, Wellard RM, et al. MR imaging of epilepsy: State of the art at 1.5T and potential of 3T. Epilepsy Dis 2003;5:3-20.

28 Briellmann RS, Jackson GD, Torn-Broers Y, et al. Causes of epilepsies: Insights from discordant monozygous twins. Ann Neurol 2001;49:45-52.

29 Briellmann RS, Berkovic SF, Jackson GD. Men may be more vulnerable to seizure associated brain damage. Neurology 2000;55:1479-85.

30 Watson C, Andermann F, Gloor P, et al. Anatomic basis of amygdaloid and hippocampal volume measurement by magnetic resonance imaging. Neurology 1992:42:1743-50

31 Briellmann RS, Jackson GD, Pell GS, et al. Structural abnormalities remote from the seizure focus: a study using $\mathrm{T} 2$ relaxometry at $3 \mathrm{~T}$. Neurology 2004;63:2303-8

32 Baxendale SA, Thompson PJ, Duncan JS. Epilepsy and depression: the effects of comorbidity on hippocampal volume-a pilot study. Seizure 2005;14:435-8.

33 Bernasconi N, Bernasconi A, Caramanos Z, et al. Mesial temporal damage in temporal lobe epilepsy: a volumetric MRI study of the hippocampus, amygdala and parahippocampal region. Brain 2003;126:462-9.
34 Bernasconi N, Duchesne S, Janke A, et al. Whole-brain voxel-based statistical analysis of gray matter and white matter in temporal lobe epilepsy. Neuroimage 2004;23:717-23

35 Marsh L, Morrell MJ, Shear PK, et al. Cortical and hippocampal volume deficits in temporal lobe epilepsy. Epilepsia 1997;38:576-87.

36 Mitchell LA Jackson GD, Kalnins RM et al. Anterior temporal abnormality in temporal lobe epilepsy: a quantitative MRI and histopathologic study. Neurology 1999;52:327-36

37 Van Paesschen W, Revesz T, Duncan JS, et al. Quantitative neuropathology and quantitative magnetic resonance imaging of the hippocampus in temporal lobe epilepsy. Ann Neurol 1997;42:756-66.

38 Briellmann RS, Kalnins RM, Berkovic SF, et al. Hippocampal pathology in refractory TLE: T2-weighted signal change reflects dentate gliosis. Neurology 2002;58:265-71

39 Wieshmann UC, Woermann FG, Lemieeux L, et al. Development of hippocampal atrophy: a serial magnetic resonance imaging study in a patient who developed epilepsy after generalized status epilepticus. Epilepsia 1997;38:1238-41.

40 Abou-Khalil B, Andermann E, Andermann F, et al. Temporal lobe epilepsy after prolonged febrile convulsions: Excellent outcome after surgical treatment. Epilepsia 1993:34:878-83.

41 Fernàndez $G$, Effenberger $O$, Vinz $B$, et al. Hippocampal malformation as a cause of familial febrile convulsions and subsequent hippocampal sclerosis. Neurology 1998;50:909-17.

42 Berkovic SF, Jackson GD. The hippocampal sclerosis whodunit: enter the genes. Ann Neurol 2000;47:557-8.

43 Jackson GD, VanPaesschen W. Hippocampal sclerosis in the MR era. Epilepsia 2002:43(Suppl 1), 4-10.

44 Satishchandra P, Krishnamoorthy ES, van Elst LT, et al. Mesial temporal structures and comorbid anxiety in refractory partial epilepsy. J Neuropsychiatry Clin Neurosci 2003;15:450-2.

45 Tebartz van Elst L, Woermann F, Lemieux L, et al. Increased amygdala volumes in female and depressed humans. A quantitative magnetic resonance imaging study. Neurosci Lett 2000;28:103-6.

46 Surguladze S, Brammer MJ, Keedwell $P$, et al. A differential pattern of neural response toward sad versus happy facial expressions in major depressive disorder. Biol Psychiatry 2005;57:201-9.

47 Hamidi M, Drevets WC, Price JL. Glial reduction in amygdala in major depressive disorder is due to oligodendrocytes. Biol Psychiatry 2004;55:563-9.

\section{bmjupdates+}

bmjupdates+ is a unique and free alerting service, designed to keep you up to date with the medical literature that is truly important to your practice.

bmjupdates+ will alert you to important new research and will provide you with the best new evidence concerning important advances in health care, tailored to your medical interests and time demands.

Where does the information come from?

bmjupdates+ applies an expert critical appraisal filter to over 100 top medical journals A panel of over 2000 physicians find the few 'must read' studies for each area of clinical interest

Sign up to receive your tailored email alerts, searching access and more...

www.bmjupdates.com 